apuntesuniversitarios.upeu.edu.pe

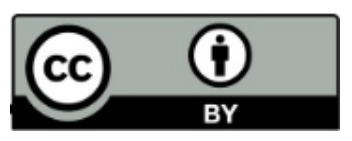

$C C-B Y$

Apuntes Universitarios, 2021: 11(3), julio-setiembre

ISSN: 2304-0335 DOI:https://doi.org/10.17162/au.v11i3.703

\title{
La imagen institucional y el cumplimiento misional como predictores de la satisfacción estudiantil
}

\section{Institutional image and missionary development as predictors of school satisfaction}

\author{
Oscar Andrés Pereira Álvarez ${ }^{1 \mathrm{a}}$ \\ Universidad de Montemorelos Mexico ${ }^{1}$
}

D Orcid ID: https://orcid.org/0000-0001-5936-1327

Recibido: 04 de enero de 2020

Aceptado: 28 de marzo de 2021

\begin{abstract}
Resumen
Las variables imagen institucional y el cumplimiento misional como predictores de la satisfacción estudiantil, hacen parte de una investigación cuantitativa de tipo correlacional, aplicada a 352 estudiantes de las 20 Instituciones Educativas Adventistas de la Unión Colombiana del Sur. El propósito es conocer la correlación existente entre la satisfacción del estudiante como receptor de los servicios del sistema educativo adventistas, y las variables de imagen institucional y cumplimiento misional, para efectos de fidelización y retención de los estudiantes actuales. Se realizó un análisis de regresión lineal múltiple por el método de pasos sucesivos, mediante el cual se encontró que las variables, percepción de la imagen institucional y percepción del cumplimiento misional explican un $47.9 \%$ la varianza de la satisfacción estudiantil.
\end{abstract}

Palabras clave: Misión; percepción; satisfacción estudiantil; imagen.

\begin{abstract}
The variables institutional image and missionary fulfillment as predictors of student satisfaction are part of a correlational quantitative investigation, applied to 163 students from the 20 Adventist Educational Institutions of the South Colombian Union. The purpose is to know the correlation between student satisfaction as a recipient of the services of the Adventist educational system, and the variables of institutional image and missionary fulfillment, for the purposes of loyalty and retention of current students. A linear regression analysis was carried out by the method of successive steps, by which it was found that the variables, perception of the institutional image and perception of missionary fulfillment explain $47.9 \%$ the variance of student satisfaction.
\end{abstract}

${ }^{\mathrm{a} C}$ Correspondencia al autor:

email: opereira@unac.edu.co 
Keywords: Mission; perception; image; student satisfaction.

\section{Introducción}

Las instituciones educativas adventistas son centros de influencia cristiana, White (2009) las define como centros de evangelismo y conversión de las personas al cristianismo. Tienen la finalidad de educar a las personas para la vida actual y futura. Por tanto, las Instituciones Educativas Adventistas de la Unión Colombiana del Sur generan en sus comunidades educativas principios, valores y aptitudes cristianas a través de sus procesos formativos como lo menciona Grajales Guerra y León Vásquez (2007) al referirse a la educación cristiana como un medio para generar un impacto de fe en todos los miembros de una fe. Para generarse la educación debe satisfacer a los estudiantes, debe suplir las expectativas y necesidades de los receptores del servicio educativo, desde este enfoque Sánchez Quintero (2018) manifiesta que solo a través de la satisfacción del estudiante se puede tener éxito en el mercado escolar, se garantiza la supervivencia de la institución y se genera una mayor demanda del servicio educativo.

La imagen institucional, la satisfacción estudiantil y la misión institucional son factores claves para el desarrollo y progreso de una institución, según Surdez Pérez et al., (2018) estás variables infieren en el concepto de satisfacción de los estudiantes. Entiendo la imagen institucional como un fenómeno mental que reúne todas las experiencias y percepciones del sujeto en relación con una realidad (Grajales Guerra y León Vásquez, 2007), la misión institucional es la razón de ser de una empresa (Maragno Coral et al., 2014) y la satisfacción del estudiante la capacidad de la institución de suplir las expectativas de los estudiantes en lo referente al servicio escolar (Sánchez Quintero, 2018).

La regresión múltiple tiene cuatro supuestos importantes que deben seguirse para hacer un análisis preciso de los cuales habla (Díaz, 1995)al apoyar la actividad y mencionar que este tipo de análisis busca relacionar múltiples variables de intervalo o nominales (variables predictoras) con otra variable más (variable criterio). Para esta investigación se tomó como enfoque principal este modelo, ya que permite analizar cuál es la relación que tienen las variables que se mencionaron y se definieron anteriormente. Por lo que se puede 
entender cómo influye la imagen institucional y el cumplimiento misional en la satisfacción del cliente en un contexto educativo.

El propósito de la presente investigación es generar calidad educativa a través de la satisfacción de los estudiantes, como menciona Cadena-Badilla et al. (2015) al hablar de calidad como uno de los mejores medios para suplir las necesidades de los estudiantes, teniendo como referente la misión institucional, ya que según Fred (2003) la misión institucional es el fundamento de prioridades, planes, estrategias y tareas. Se definieron cada uno de los elementos claves, buscando diferentes fuentes y conceptos que permitieron tener una visión amplia y variada acerca de cada una de ellas. Después de esto se realizó la relación que existe entre estas variables.

Para el análisis de la hipótesis principal se utilizó la técnica estadística análisis de regresión lineal múltiple, la cual consiste en calcular la relación entre variables por medio de un ajuste matemático. El objetivo de esta investigación fue analizar si las variables misión institucional e imagen institucional son predictores significativos de la satisfacción del cliente, según la percepción de los estudiantes del sistema educativo de la Unión Colombiana del Sur. En la Figura 1 se muestra el modelo teórico propuesto.

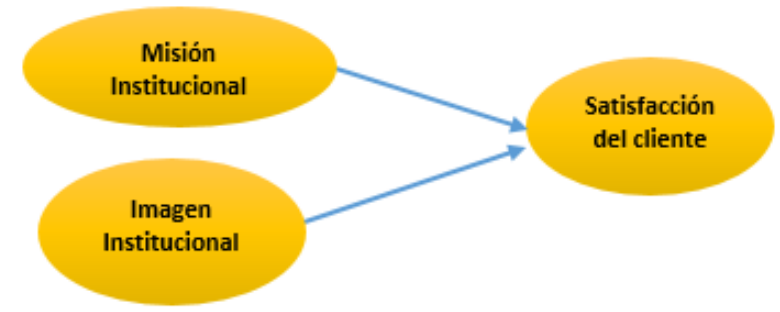

Figura 1. Modelo teórico propuesto.

Fuente: Elaboración propia.

\section{Revisión literaria}

La primera variable es la percepción de la imagen institucional, definida por Duque Oliva y Carvajal Prieto (2015) como la impresión generada en la mente del público debido a la interacción de sus creencias, experiencias y sentimientos al relacionarse directa o indirectamente con un producto o servicio. Para Klinger et al., (2014) esta impresión mental o valorización generada en el cliente, solo puede producirse por la experiencia o los sentidos del cliente, al relacionarse con el producto o servicio. Para Dumont et al., (2018) la imagen 
institucional puede representarse como el signo de identidad, la firma de una empresa o un activo intangible, generando una expectativa en el cliente. Esta expectativa según Guédez y Osta (2012) lleva al cliente a realizar una valoración racional y emocional de los atributos tangibles e intangibles de la institución.

La segunda variable del estudio, es la percepción del cumplimiento de la misión. Para Hax y Majluf (1997) es la esencia de lo que una empresa es en realidad, sobre la cual se fundamentan los planes, prioridades, estrategias y tareas. Según Cardona (2015) es la razón de ser de la empresa, la encargada de dar sentido a su existencia, es el horizonte que traza el camino por donde todos los empleados deben seguir y vincula a todo el personal bajo una misma meta y objetivos generando una identidad. Ballvé y Debeljuh (2006) afirma que la misión institucional es la que genera la identidad de la empresa y define el carácter perdurable de la organización. Es el objetivo supremo y permanente que señala la razón de existencia de la empresa, su propósito a alcanzar. (Mejía y López, 2016).

La tercera y última variable de investigación, es la satisfacción del estudiante. Para Surdez Pérez et al., (2018) se puede entender al estudiante como un ser con prejuicios de valor dispuesto a comparar un bien o servicio con la imagen generada antes de su compra. Álvarez et al., (2015) define la satisfacción estudiantil como la sensación de bienestar que experimenta el estudiante al sentir satisfecha sus expectativas. En este sentido González et al., (2011) manifiesta que para satisfacer al estudiante se debe ser eficiente en calidad académica. Hanns et al., (2010) agrega que la satisfacción del estudiante está relacionado con la calidad de la misión y con la reputación o imagen de la institución, para Gento Palacios y Vivas García (2003) las instituciones educativas que satisfacen a los estudiantes reducen la deserción escolar, se vuelven competitivas y mejoran el rendimiento académico de los estudiantes y Gómez et al., (2013) señala que la satisfacción es un indicador de la calidad académica.

Pamies (2004) al hablar de la satisfacción estudiantil utiliza el concepto de disparidad del valor percibido, debido a que se toma en cuenta el bienestar emocional generado debido a la utilidad del servicio y es directamente proporcionado con su valor económico. Es un proceso que involucra las experiencias del estudiante desde el momento en que se genera la expectativa por los anuncios del servicio hasta el momento en el que se consume. Para Kotler 
y Keller (2012) este proceso también vincula las expectativas de los estudiantes, la utilidad del servicio, la relación costo - beneficio, la experiencia del estudiante y con el servicio.

\section{Sustento teórico del modelo}

Meza Escobar (1996) realizó una investigación para conocer la percepción de la imagen institucional de la Universidad de Montemorelos, México, con una muestra aleatoria y representativa de 669 estudiantes, 162 padres, 21 miembros de iglesia y 91 administrativos y docentes. La investigación concluyó que la imagen percibida por el público en general fue regularmente positiva y que la percepción positiva de los estudiantes es más baja que la de los egresados, lo cual influye en la percepción del servicio recibido.

Por otro lado, Maragno Coral et al., (1981) realizó una investigación utilizando la variable de misión institucional como elemento clave para estimular y guiar la búsqueda de oportunidades, en las empresas hoteleras de Santa Catarina, Brasil. Los resultados mostraron que ninguna empresa de la muestra posee una misión efectiva, estas utilizan la misión institucional más como instrumento de marketing que como herramienta de gestión estratégica.

Reyes Hernandéz (2014) en un estudio experimental, con una muestra de 100 clientes y 12 empleados encontró que la satisfacción del cliente fue calificada en un 63\%, 68\% por el parqueo, 70\% instalaciones, 71\% limpieza en general y 59\% capacidad del personal y el 73\% de los clientes externos perciben la calidad del servicio de una forma aceptable.

\section{Metodología}

Esta fue una investigación cuantitativa porque, según Hernández Sampieri et al. (2010), ofrece la posibilidad de generalizar los resultados más ampliamente, otorga control sobre los fenómenos, permitiendo detectar tendencias, así como un punto de vista de conteo, las magnitudes de éstos y facilita la comparación entre estudios similares con la finalidad de probar hipótesis y es de enfoque predictivo porque según Sneiderman (2006) proyecta situaciones futuras y va seguida de una exploración, un análisis y una explicación.

La población estuvo formada por 4.114 estudiantes de las 20 escuelas y colegios que conforman el sistema educativo adventista de la Unión Colombiana del Sur entre los meses enero a marzo del 2020. Se usó un muestreo probabilístico con un nivel de confianza del 95 
\% y un margen de error del 5 \%, para obtener una muestra de 352 estudiantes entre los 15 y 20 años de edad, con respecto al sexo, el 39.2\% son hombres y el 60.8\%, mujeres.

Para la recolección de los datos utilizó la encuesta como instrumento de recolección de datos, debido que Orellana López y Sánchez Gómez (2006) manifiesta que una encuesta permite conocer las percepciones de las persones a raíz de una escala bien definida. Para la variable de imagen institucional y misión institucional se utilizó una escala tipo Likert donde el número 1 representaba el concepto pésimo y el número 5 representaba el concepto excelente, ambas encuestas fueron validadas y aplicadas por Tolentino Hernández (2016) en la investigación sobre los factores determinantes de desempeño organizacional y satisfacción validados con directivos confesionales. Cada constructo está dividido en tres dimensiones y lo conforman 30 declaraciones para imagen institucional y 23 para misión institucional. Para el constructo de satisfacción al cliente se utilizó una escala tipo Likert que va desde 1 que representa el concepto totalmente insatisfecho hasta el número 5 que representa el concepto totalmente insatisfecho, con 23 criterios. La confiabilidad del instrumento se midió por variable con su correspondiente alfa de Cronbach, resultando lo siguiente: (a) misión institucional, 0.930, (b) satisfacción del cliente, 0.898 y (c) misión institucional, 0.771.

\section{Resultados}

En la Tabla 1 se muestra la media aritmética y la desviación estándar de cada constructo mediante el análisis de las respuestas provistas por 352 estudiantes. En la tabla se observa que el constructo mejor evaluado fue la imagen institucional.

\section{Tabla 1}

Descriptivos de variables

\begin{tabular}{ccc}
\hline Constructo & Media & Desviación Estándar \\
\hline Satisfacción del cliente & 4.56 & 0.393 \\
Imagen institucional & 4.51 & 0.420 \\
Misión institucional & 4.47 & 0.330 \\
\hline
\end{tabular}

Para profundizar en el estudio sobre cómo los estudiantes trabajan de manera práctica las variables en estudio, se obtuvieron las medias aritméticas de las dimensiones (ver Tabla 
2), en donde se puede observar que la dimensión más baja de la percepción de la imagen institucional fue la calidad académica y la más alta fue el campus universitario. Para el constructo de satisfacción al cliente la dimensión más baja fue el seguimiento al cliente y la más alta fue recursos humanos. Para el constructo de percepción de la misión institucional la dimensión más baja fue la percepción del cumplimiento misional y la más alta fue la proyección institucional.

\section{Tabla 2}

Medias aritméticas por constructos

\begin{tabular}{clrr}
\hline Constructo & \multicolumn{1}{c}{ Dimensiones } & $M$ & $D E$ \\
\hline \multirow{4}{*}{ Misión Institucional } & MISproyeccion_institucional & 4,56 & 0,537 \\
& MISEmpleados_idoneos & 4,26 & 0,871 \\
& MISprestacion_servicio & 4,23 & 0,711 \\
& MISValor_insitucional & 4,38 & 0,644 \\
& MISmisión_institucional & 4,81 & 0,318 \\
\hline \multirow{3}{*}{ Satisfacción del cliente } & STPRecursos_humanos & 4,55 & 0,491 \\
& STPplanta_fisica & 4,54 & 0,593 \\
& STPAmabilidad_instituciona & 4,54 & 0,783 \\
& STPBienestar_estudiantil & 4,70 & 0,439 \\
& STPcalidad_docente & 4,50 & 0,515 \\
& STPSegumiento_cliente & 4,55 & 0,668 \\
\hline \multirow{3}{*}{ Imagen institucional } & IMGCampus_Universitario & 4,49 & 0,550 \\
& IMGCompetitividad_institucional & 4,47 & 0,587 \\
& IMGRecursos_educativos & 4,55 & 0,593 \\
& IMGBuen_servicio & 4,63 & 0,626 \\
& IMGCalidad_acedemica & 4,44 & 0,664 \\
\hline
\end{tabular}

Fuente: Elaboración propia.

\section{Supuestos de regresión múltiple}

Esta regresión lineal busca relacionar múltiples variables de intervalos o nominales (independientes) con otras variables (dependientes). Para Williams et al., (2013) y Poza Lara (2008) la regresión lineal posee cuatro supuestos importantes que se deben tomar en cuenta para desarrollar una investigación fiable, los cuales son: (a) normalidad, que refiere una distribución normal de los puntajes, (b) relación lineal, es decir debe existir una correlación entre las variables, (c) aditividad y multicolinealidad, hace referente que la variable independiente por sí sola suma a la explicación de la variable dependiente o que ambas variables independientes están relacionadas. 
Para esta investigación, el supuesto de linealidad se estudia mediante diagramas de dispersión parcial para cada variable, tomando como parámetro dependiente la satisfacción del estudiante (STP). Los gráficos de la Figura 2 muestra que la variable imagen institucional (IMG) se aproxima a una distribución lineal, mientras que la misión institucional no presenta una relación positiva respecto a la recta.

a)

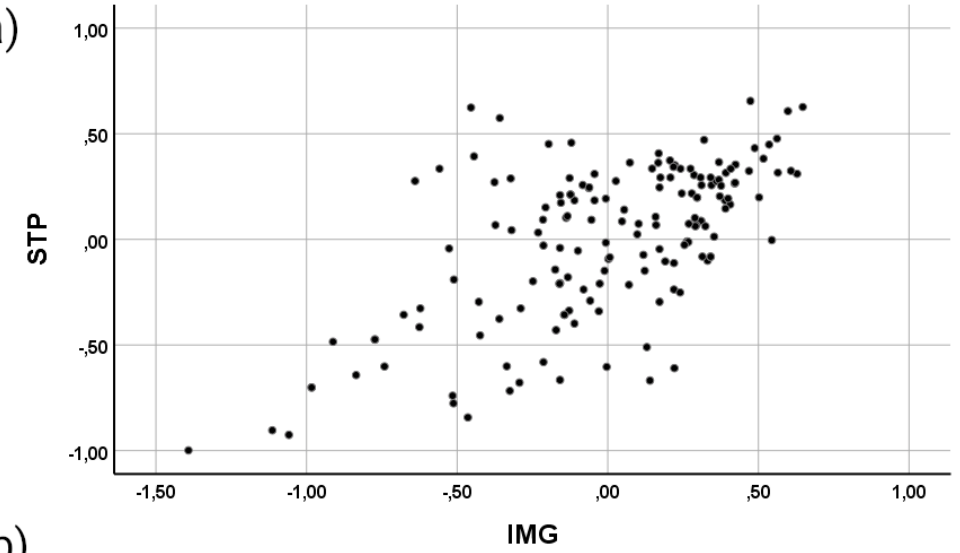

b)

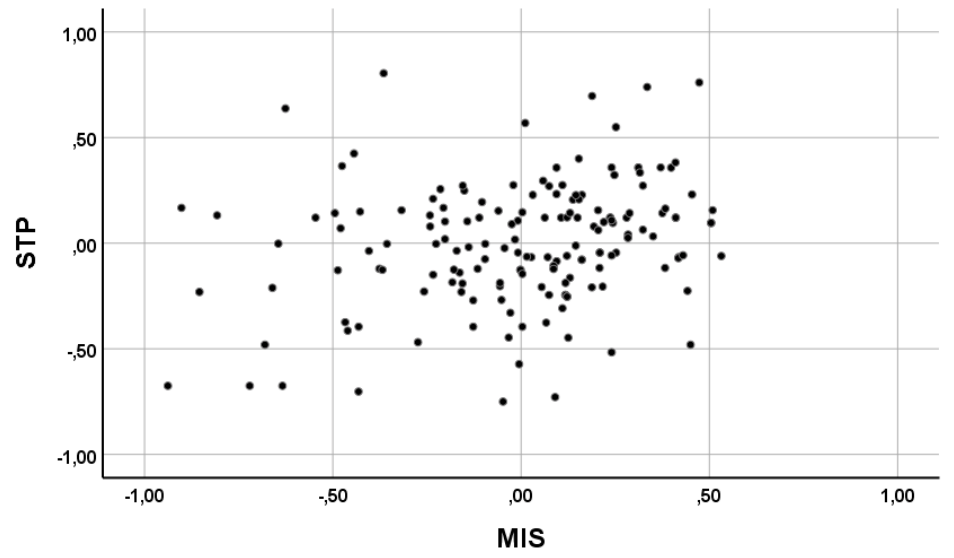

Figura 2. Linealidad con las variables propuestas.

La prueba de normalidad evalúa que las variables sigan una distribución normal. Este supuesto lo verificamos mediante métodos gráficos (Figura 3), como puede observarse en el histograma los datos denotan una distribución con forma de campana simétrica, donde la mayoría de los datos se ubican máximo a dos desviaciones estándar de la media. En el gráfico P-P normal nuestros datos se aproximan muy bien a la distribución normal, tomando como variable dependiente la satisfacción de los estudiantes (STP). Otra forma de evaluar la normalidad de los datos es con la prueba K-S (Kolmogorov-Smirnov), donde la la significación asintótica bilateral es $0.20>0.05$ por lo que se asume que sigue la ley normal. 
a)

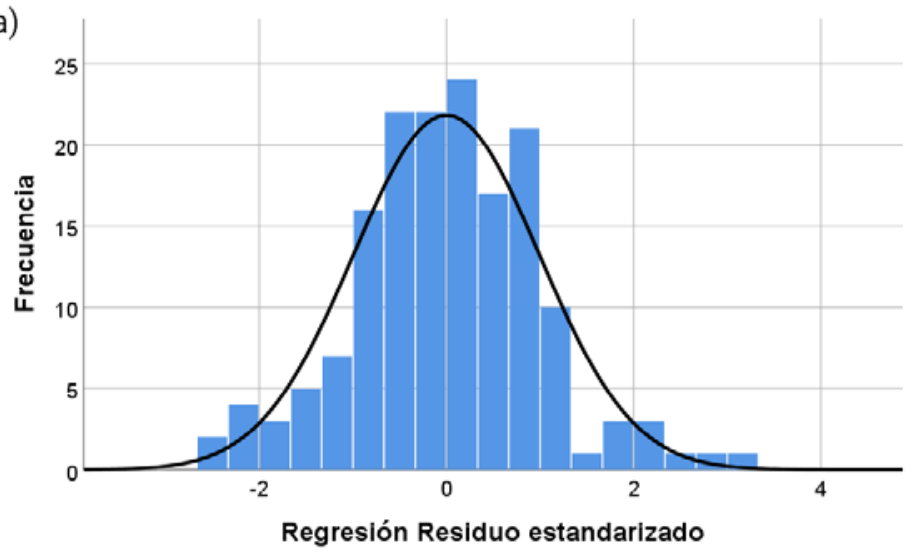

b)

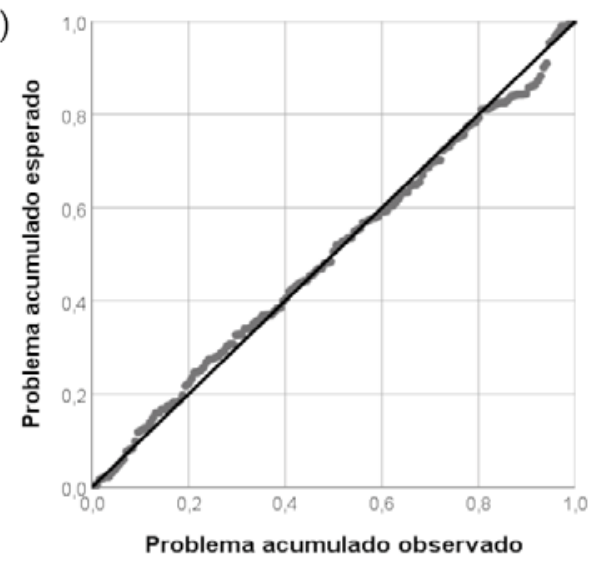

Figura 3. Análisis de normalidad de los datos. a) Histograma de residuos estandarizados de las variables, b) Gráfico P-P normal de regresión Residuo tipificado.

En el tercer criterio se probó la independencia de los errores, este busca determinar si los errores en las variables explicativas son independientes entre sí. Ante diferentes observaciones de una misma variable en el tiempo, puede darse una cierta inercia en el sistema que hace que los valores sucesivos se aproximen entre sí. Se considerarán independientes si el estadístico se encuentra entre 1.5 y 2.5, nuestro resultado de la prueba de Durbin-Watson fue $\mathrm{DW}=1.773$, lo que indica que los errores son independientes (ver Tabla 3). Para evaluar la correlación entre variables independientes se hizo un diagnóstico de la colinealidad (Tabla 4), existe multicolinealidad entre las variables cuando hay algún tipo de dependencia lineal entre ellas. Un valor de tolerancia cercano a uno y un factor de inflación de la varianza menor a 10.0 indica que no existe multicolinealidad, las variables IMG y MIS cumplen estos criterios, por lo que no están correlacionadas entre sí.

\section{Tabla 3}

Independencia de los errores con la prueba Durbin-Watson

\begin{tabular}{lrrrrr}
\hline Modelo & $\mathrm{R}$ & R cuadrado & $\begin{array}{c}\text { R cuadrado } \\
\text { corregida }\end{array}$ & $\begin{array}{c}\text { Error típ. de la } \\
\text { estimación }\end{array}$ & Durbin-Watson \\
\hline 1 & 0.698 & 0.487 & 0.480 & 0.28237 & 1.773 \\
\hline
\end{tabular}


Tabla 4

Diagnóstico de la colinealidad

\begin{tabular}{lrr}
\hline Variable & Tolerancia & \multicolumn{1}{c}{ FIV } \\
\hline IMG & 0.913 & 1.095 \\
\hline MIS & 0.852 & 1.095 \\
\hline
\end{tabular}

\section{Prueba de hipótesis nula}

$H_{0}$ La imagen institucional y el cumplimiento misional no son predictores de la percepción de satisfacción por parte de los estudiantes de las escuelas y colegios adventistas de la Unión Colombiana del Sur. Para el análisis de esta hipótesis, se utilizó la técnica estadística de regresión lineal múltiple por el método de pasos sucesivos. No se eliminó ninguna variable, se consideraron como variables independientes la percepción de la imagen institucional y la percepción del cumplimiento de la misión, y como variable dependiente, la satisfacción del estudiante. El modelo se expresa de la siguiente manera:

$$
\begin{gathered}
Y=\beta_{0}+\beta_{1} \cdot X_{1}+\beta_{2} \cdot X_{2}+\varepsilon_{1} \\
S T P=\beta_{0}+\beta_{1} \cdot I M G+\beta_{2} \cdot M I S+\varepsilon_{1}
\end{gathered}
$$

Donde:

$\mathrm{Y}=$ Satisfacción del estudiante (STP)

$X_{1}=$ Percepción de la imagen institucional (IMG)

$X_{2}=$ Percepción del cumplimiento misional (MIS)

Al realizar el análisis de regresión se encontró un valor de $\mathrm{R}^{2}$ corregida de 0.480 , lo cual indica que estas dos variables explican en un 48,0 \% la varianza de la variable dependiente satisfacción al cliente (ver Tabla 5).

\section{Tabla 5}

\begin{tabular}{|c|c|c|c|c|}
\hline Modelo & $\mathrm{R}$ & R cuadrado & $\begin{array}{c}\text { R cuadrado } \\
\text { corregida }\end{array}$ & Error típ. de la estimación \\
\hline 1 & 0.698 & 0.487 & 0.480 & 0.28237 \\
\hline
\end{tabular}

Resumen del modelo

En la Tabla 6 se muestran los valores de los coeficientes estandarizados, se encontró que la imagen institucional y la misión institucional fueron predictores significativos de la satisfacción del cliente. El mejor predictor fue la imagen institucional ( $\beta=0.613)$, por lo tanto, se retiene la hipótesis de investigación y se rechaza la hipótesis nula. 
Tabla 6

Coeficientes

\begin{tabular}{|c|c|c|c|c|c|c|}
\hline \multirow[b]{2}{*}{ Modelc } & & \multicolumn{2}{|c|}{$\begin{array}{l}\text { Coeficientes no } \\
\text { estandarizados }\end{array}$} & $\begin{array}{c}\text { Coeficientes } \\
\text { estandarizados }\end{array}$ & \multirow[b]{2}{*}{$\mathrm{t}$} & \multirow[b]{2}{*}{ Sig. } \\
\hline & & $\mathrm{B}$ & Error típ. & Beta & & \\
\hline \multirow[t]{3}{*}{1} & (Constante) & .883 & .343 & & 2.576 & .011 \\
\hline & Imagen & .585 & .057 & .613 & 10.339 & .000 \\
\hline & Misión & .234 & .070 & .197 & 3.319 & .001 \\
\hline
\end{tabular}

Para calcular el tamaño del efecto de la regresión se utilizó el G*Power que es un programa estadístico, de descarga gratuita, diseñado para realizar estimaciones de la potencia estadística y del tamaño del efecto. Se hizo un análisis post hoc, ya que se había aplicado las encuestas y para calcular el tamaño del efecto en regresiones se utilizó la siguiente fórmula:

$$
\mathrm{f}^{2}=\frac{R^{2}}{1-R^{2}}
$$

Se obtuvo un coeficiente de determinación de $R^{2}$ de 0.486 . Al calcular el tamaño del efecto se encontró un valor de 0.945. Este valor es considerado como una medida del tamaño del efecto muy alto (> .35). Al calcular la potencia estadística se encontró un valor de 1.0. La potencia estadística $(1-\beta=1.0)$ supera los niveles mínimos exigidos $(80 \%)$, por lo tanto, la potencia estadística se considera como muy alta.

\section{Discusión}

Teniendo en cuenta los diferentes análisis que se han podido realizar de manera formal e informal, se pretendió por medio de esta investigación dar una respuesta al interrogante: ¿Cuál es el nivel de contribución de la satisfacción del cliente y la calidad del servicio en los colegios pertenecientes al sistema educativo de la Unión Colombiana del Sur sobre la competitividad del mercado educativo en su entorno? Tomando como base la pregunta de investigación, los resultados dados plantean y dan a conocer que los tres elementos principales de esta investigación, los cuales se han mencionado anteriormente (La imagen institucional, la satisfacción del cliente y la misión institucional), guardan una interrelación. Esto permite proponer que la institución puede posicionarse en la 
competitividad educativa mediante un desarrollo adecuado de la imagen y la misión institucional.

Los resultados obtenidos tienen coherencia con los resultados registrados en otras investigaciones similares, donde su foco principal ha sido el saber cuál es la importancia de la satisfacción del cliente y cómo se logra. Por lo tanto, vale la pena mencionar que Cahuana Sáncheza (2018) realizó una investigación en la Unión Colombiana del Norte donde tuvo en cuenta dos variables: la satisfacción docente y la calidad del servicio, encontrando una correlación positiva entre estos dos factores. De esta forma podemos concluir que, al tener una correlación tan directa e importante, es de forma indispensable darle una importancia mayor al momento de dirigir una empresa o en este caso una institución educativa.

\section{Conclusión}

Esta investigación demostró que la percepción de la imagen institucional y su misión tienen una correlación con la satisfacción del cliente. Aunque la imagen institucional tuvo una mayor capacidad predictiva en lograr la satisfacción de los estudiantes ante el servicio prestado por los colegios adventistas de la Unión Colombiana del Sur. Este hallazgo es relevante, ya que puede aportar información a los administradores para que sigan invirtiendo en la promoción y evaluación de la imagen institucional, como una garantía de la satisfacción de sus clientes y de la competitividad en el mercado.

La Organización Internacional para la Normalización ISO (2005) señala que las instituciones que penden únicamente de los clientes deben esforzarse en cumplir sus expectativas para poder fidelizarlos, desde este enfoque Surdez Pérez et al., (2018) señala que la supervivencia de la institución depende en gran medida de la capacidad de la misma por suplir las expectativas de los estudiantes frente a los servicios ofrecidos por la institución, dentro de los servicios Bartolomé et al., (2006) señala como de gran importancia la eficacia administrativa, el cuerpo docente, la infraestructura y el reconocimiento de la institución o el estatus de su imagen. De estos servicios Hanns et al., (2010) señala que la misión institucional y la imagen institucional son referentes que intervienen en la sensación de bienestar y satisfacción que experimentan los estudiantes en la instituciones educativas. 


\section{Referencias}

Álvarez, J., Chaparro, E., \& Reyes, D. (2015). Estudio de la satisfaccion de estudiantes con servicios educativos brindados por instituciones de educ superior. REICE: Revista Electrónica Iberoamericana Sobre Calidad, Eficacia y Cambio En Educación, 13(2), 5-26. https://revistas.uam.es/index.php/reice/article/view/2788

Ballvé, A. M., \& Debeljuh, P. (2006). Misión y valores. La empresa en busca de su sentido. Buenos Aires: Grupo Editorial Planeta.

Bartolomé, A., Blanco, O., Hernández, R., y Murguey, V. (2006). Calidad en la enseñanza universitaria (una experiencia institucional). Geoenseñanza, 11(2), 163-173. https://www.redalyc.org/articulo.oa?id=36012425003

Cahuana Sáncheza, J. H. (2018). Calidad de los servicios educativos y la satisfacción de los clientes de la Universidad Nacional Mayor de San Marcos. Revista Muro de La Investigación, 1(2). https://doi.org/10.17162/rmi.v1i2.758

Cardona, M. S. (2015). Educación para la cultura de la paz. Una aproximación psicopedagógica. Ediciones USTA.

Díaz, F. (1995). La predicción del rendimiento académico en la universidad: un ejemplo de aplicación. Enseñanza, 13, 43-61. https://revistas.usal.es/index.php/02125374/article/view/4054

Dumont, J. R. D., Lira, L. A. N., \& Limaco, K. V. C. (2018). Influencia de las competencias gerenciales y la gestión por resultados en la imagen institucional. Fides et RatioRevista de Difusión Cultural y Científica de La Universidad La Salle En Bolivia, 16(6), 169-197. http://www.scielo.org.bo/scielo.php?script=sci_abstract\&pid=S2071081X2018000200010\&lng=pt\&nrm=iso

Duque Oliva, E. J., y Carvajal Prieto, L. A. (2015). La identidad organizacional y su influencia en la imagen: una reflexión teórica - Organizational identity and its influence on image: A theoretical reflection. Suma de Negocios, 6(13), 114-123. https://doi.org/10.1016/j.sumneg.2015.08.011

Fred, D. (2003). Conceptos de administración estrategica. México: Pearson.

Gento Palacios, S., y Vivas García, M. (2003). El SEUE: un instrumento para conocer la satisfacción de los estudiantes universitarios con su educación. Acción Pedagógica, 12(2), 16-27. https://dialnet.unirioja.es/servlet/articulo?codigo=2972060

Gómez, S., López, M., Reyes, R., y Gama, L. (2013). Lealtad, satisfacción y rendimiento académico en los estudiantes de la uaslp-uamzm - Dialnet. Revista De Investigaciones. https://revistas.ugca.edu.co/index.php/sophia/article/view/55

González, A. J., Carrillo, B. T., y Robles, J. (2011). Evaluación de la satisfacción académica 
de los estudiantes de la Universidad Autónoma de Nayarit. Revista Fuente 3(6), 4656. http://dspace.uan.mx:8080/jspui/handle/123456789/1431

Grajales Guerra, T., y León Vásquez, V. (2007). Importancia y efectividad de la filosofía educativa adventista en Chile. RIEE | Revista Internacional de Estudios En Educación, 7(2), 82-94. https://doi.org/10.37354/riee.2007.072

Guédez, C., y Osta, K. (2012). Factores de la Imagen Institucional Universitaria: perspectiva desde un sector del público interno, personal administrativo. Revista Ingeniería Industrial, 11, 71-84. http://revistas.ubiobio.cl/index.php/RI/article/view/28

Hanns, de la F. M., Mercedes, M. N., \& María Jesús, R. R. (2010). Análisis De La Satisfacción Universitaria En La Facultad De Ingeniería De La Universidad De Talca / Analysis of Satisfaction of Students of the Faculty of Engineering of Universidad De Talca. Ingeniare. Revista Chilena de Ingeniería VO - 18, 18(3), 350. http://dx.doi.org/10.4067/S0718-33052010000300009.

Hax, A., y Majluf, N. (1997). Estrategias para el liderazgo competitivo (Granica). Buenos Aires: Granica.

Hernández Sampieri, R., Fernández Collados, C., \& Del Pilar Baptita, M. (2010). Metodología de la investigación (Sexta). México: Interamericanada de editores.

ISO. (2005). sistema de gestión de la calidad - Fundamentos y vocabulario. Normativa ISO, $2005,42$.

Klinger, R., Olaya, J., Canaval, J., Giraldo, A., Mosquera, J., \& Bermudez, M. (2014). Medición de la imagen institucional de una universidad entre estudiantes de último año de educación secundaria. Sophia, 10(1), 83-94. https://revistas.ugca.edu.co/index.php/sophia/article/view/220

Kotler, P., y Keller, K. L. (2012). Información del beneficiario. México: Pearson Education.

Maragno Coral, S., de Souza, P., \& Jao Lunkes, R. (1981). Misión institucional: Análisis de los principales elementos propuestos por Pearce II (1982) presentes en las misiones de los hoteles de Santa Catarina, Brasil. Journal of Chemical Information and Modeling, 53(9), 1689-1699.

Maragno Coral, S., de Souza, P., y Lunkes, R. (2014). Misión institucional: Análisis de los principales elementos propuestos por Pearce II (1982) presentes en las misiones de los hoteles de Santa Catarina, Brasil. Estudios y Perspectivas En Turismo, 23(3), 467483. https://www.redalyc.org/articulo.oa?id=180731336003

Martín, C. V., Agustín, M. A., Arturo, V. R., y Joaquin, V. Q. (2015). La satisfacción estudiantil universitaria: análisis estratégico a partir del análisis de factores. Industrial Data, 18(1), 9-18. https://doi.org/10.15381/idata.v18i1.12062

Mejía, J. F., \& López, D. (2016). Modelo de Calidad de E-learning para Instituciones de 
Educación Superior en Colombia E-learning Quality Model for Higher Education Institutions in Colombia. Formación Universitaria, 9(2), 59-72. https://doi.org/10.4067/S0718-50062016000200007

Meza Escobar, M. (1996). Imagen institucional de la Universidad de Montemorelos percibida por públicos selectos. Tesis de doctorado. Universidad de Montemorelos. http://dspace.biblioteca.um.edu.mx/xmlui/handle/20.500.11972/753

Orellana López, D., y Sánchez Gómez, M. (2006). Técnicas de recolección de datos en entornos virtuales más usadas en la investigación cualitativa. Revista de Investigación Educativa, RIE, 24(1), 205-222. https://revistas.um.es/rie/article/view/97661

Pamies, D. S. (2004). De la calidad de servicio a la fidelidad del cliente. Madrid: ESIC.

Poza Lara, C. (2008). Técnicas estadísticas multivariantes para la generación de variables latentes. Revista Escuela de Administración de Negocios, 64(0120-8160), 89-99.

Reyes Hernandéz, S. P. (2014). Calidad del servicio para aunmentar la satisfacción del cliente de la asociaciom Share, sede huehuetenango. Tesis de grado. Universidad Rafael Landívar. http://biblio3.url.edu.gt/Tesario/2014/01/01/Reyes-Sonia.pdf

Sánchez Quintero, J. (2018). Satisfacción estudiantil en educación superior: validez de su medición. Bogota: Sergio Arboleda.

Sneiderman, S. (2006). Las tecnicas proyectivas como metodo de investigacion y diagnostico. actualizacion en tecnicas verbales: “el cuestionario desiderativo." Subjetividad $\quad y \quad$ Procesos Cognitivos, 8, 296-331. http://www.redalyc.org/articulo.oa?id=339630247014\%3E ISSN 1666-244X

Surdez Pérez, E. G., Sandoval Caraveo, M. del C., y Lamoyi, C. L. (2018). Satisfacción estudiantil en la valoración de la calidad educativa universitaria. Educación y Educadores, 21(1), 9-26. https://doi.org/10.5294/edu.2018.21.1.1

Tolentino Hernández, M. H. (2016). Factores determinantes de desempeño organizacional y satisfacción validados con directivos confesionales. Tesis doctoral. Universidad de Montemorelos. http://dspace.biblioteca.um.edu.mx/xmlui/handle/20.500.11972/199

White, E. (2009). La educación cristiana. Buenos Aires: ACES.

Williams, M., Alberto, C., y Grajales, G. (2013). Assumptions of Multiple Regression: Correcting Two Misconceptions - Practical Assessment, Research \& Evaluation. Evaluación Práctica, Investigación y Evaluación, 18(11). https://doi.org/10.7275/55hn-wk47 